\title{
MIXTURE KALMAN FILTERING FOR JOINT CARRIER RECOVERY AND CHANNEL ESTIMATION IN TIME-SELECTIVE RAYLEIGH FADING CHANNELS
}

\author{
Ali A. Nasir, Salman Durrani and Rodney A. Kennedy \\ School of Engineering, CECS, The Australian National University, Canberra, Australia. \\ Email: \{ali.nasir, salman.durrani, rodney.kennedy\}@anu.edu.au
}

\begin{abstract}
This paper proposes a new blind algorithm, based on Mixture Kalman Filtering (MKF), for joint carrier recovery and channel estimation in time-selective Rayleigh fading channels. MKF is a powerful tool for estimating unknown parameters in non-linear, non-Gaussian, real-time applications. We use a combination of Kalman filtering and Sequential Monte Carlo Sampling to estimate the channel fading coefficients and joint posterior probability density of the unknown carrier offset and transmitted data respectively. We study the effect of Signal to Noise Ratio (SNR) and doppler shift on Mean Square Error (MSE) and Bit Error Rate (BER) performance of the proposed algorithm through computer simulations. The results show that BER of the proposed algorithm achieves the theoretical performance slope for the full acquisition range of normalized carrier frequency offset.
\end{abstract}

Index Terms - Synchronization, Mixture Kalman Filtering, Sequential Importance Sampling, Frequency offset, Channel Estimation estimation.

\section{INTRODUCTION}

In mobile wireless communication channels, loss of synchronization may occur due to Carrier Frequency Offset (CFO) and/or Doppler effects. The CFOs arise due to the mismatch between transmitter and receiver oscillators. Its correction is a fundamental requirement for reliable data transmission in any wireless communication system [1]. Mitigation of such offsets becomes more sensitive in time-selective fading channels.

The existing CFO estimators in fading channels can be classified as either data-aided $[2,3]$ or blind $[1,4,5]$. Data aided schemes use training or pilot symbols and are not bandwidth efficient [5]. Blind schemes do not require any initial training sequences. Existing blind CFO estimators exploit cyclostationary statistics of the received signal $[1,4]$ or use transmitter-induced cyclostationary precoders [5]. These algorithms assume either oversampling of the received signal [4] or large number of symbols in the transmission frame [1,4] or work under limited range of frequency offset estimation $[1,4,5]$.

There has been growing interest in the application of Mixture Kalman Filtering (MKF) to different problems in wireless communication systems $[6,7]$. MKF is a combination of Kalman filtering and Sequential Monte Carlo Sampling techniques (also referred to as particle filtering methods) [7]. MKF draws Monte Carlo samples only in the indicator space and uses a mixture of Gaussian distributions to approximate the target distribution [6]. Prior work in this area has looked at problems for joint channel estimation and data detection [6] and joint blind timing estimation and data detection [7], respectively. To the best of author's knowledge, the use of MKF for joint carrier frequency offset estimation and channel tracking in time-selective Rayleigh fading channels has not been considered before.

In this paper, we propose a combination of Kalman filtering and Sequential Importance Sampling (a popular particle filtering technique) to estimate the channel fading coefficients and joint posterior probability density of the unknown carrier offset and transmitted data respectively. We adapt the parameter estimates using symbol by symbol processing, which does not require long transmission frames. We demonstrate robust Bit Error Rate (BER) and Mean Square Error (MSE) performance of the proposed algorithm for fullrange acquisition of carrier frequency offset $|f|<1 / 2$, without the need for oversampling of the received signal. Moreover, the BER of our proposed algorithm achieves the theoretical performance slope for all values of SNR.

\section{SYSTEM MODEL}

\subsection{Received Signal}

We consider a digital communication system where the transmitter sends the data symbols, chosen from a discrete alphabet of size $M$, over time-selective Rayleigh fading channel. The $k^{\text {th }}$ sample of complex-baseband received sequence, $y_{k}$, can be written as

$$
y_{k}=h_{k} e^{j 2 \pi f k} s_{k}+w_{k},
$$

where $h_{k}$ is the $k^{\text {th }}$ complex multiplicative noise introduced by the frequency-flat Rayleigh fading channel, $w_{k}$ is the Additive White Gaussian Noise (AWGN), $s_{k} \in \mathcal{S}$, is the $k^{\text {th }}$ transmitted symbol, $k=\{0,1, \ldots, K-1\}, K$ is the frame length and $f$ is the digital frequency offset between transmitter and receiver oscillators, in cycles/sample. $\mathcal{S}$ belongs to the set of possible transmitted symbols $\mathcal{S}=\left\{S_{0}, S_{1}, \ldots, S_{M-1}\right\}$ for $M$-PSK constellation.

The variation of the fading coefficient $h_{k}$ can be modeled by a second order autoregression driven by a complex white Gaussian process [8]

$$
h_{k}=\beta_{1} h_{k-1}+\beta_{2} h_{k-2}+v_{k},
$$

where the values of the coefficients of the autoregressive process $\beta_{1}$ and $\beta_{2}$ and the variance of the zero-mean complex white Gaussian noise $v_{k}$ are functions of the fading rate of the channel [7]. The channel model in (2) can be expressed as

$$
\mathbf{h}_{k}=\mathbf{B} \mathbf{h}_{k-1}+\mathbf{c} v_{k},
$$

where

$$
\mathbf{B}=\left[\begin{array}{cc}
\beta_{1} & \beta_{2} \\
1 & 0
\end{array}\right],
$$

$\mathbf{h}_{k}=\left[h_{k}, h_{k-1}\right]^{T}, \mathbf{c}=[1,0]^{T}$ and $(\cdot)^{T}$ denotes the transpose operator. Thus, received sequence in (1) can be written as

$$
y_{k}=\mathbf{c}^{T} \mathbf{h}_{k} e^{j 2 \pi f k} s_{k}+w_{k},
$$




\subsection{Estimation Objective}

The general objective is to jointly estimate the transmitted symbols $s_{0: K-1}=\left\{s_{0}, s_{1}, \ldots, s_{K-1}\right\}$, the complex fading coefficients $h_{0: K-1}=\left\{h_{0}, h_{1}, \ldots, h_{K-1}\right\}$ and the static frequency offset $f$, using the received sequence $y_{0: K-1}=\left\{y_{0}, y_{1}, \ldots, y_{K-1}\right\}$.

\section{PROPOSED ALGORITHM ( MIXTURE KALMAN FILTERING )}

We use Mixture Kalman Filtering (MKF) based algorithm to estimate the system state $\left(s_{k}, \mathbf{h}_{k}, f\right)$ at time $k$. We handle the fading coefficients, $\mathbf{h}_{k}$, using Kalman filtering and update the reduced systems state, $\left(s_{k}, f\right)$ using a popular particle filtering technique, known as Sequential Importance Sampling (SIS). According to the Bayesian perspective, all the necessary information for the estimation of unknown parameters $\left(s_{k}, f\right)$ at time $k$, is contained in the joint posterior probability distribution function (pdf) $p\left(s_{0: k}, f \mid y_{0: k}\right)$. The particle filter approximates the posterior pdf of unknown variables, $p\left(s_{0: k}, f \mid y_{0: k}\right)$, by a set of $N$ particles, $\left\{\left(s_{0: k}, f_{0: k}\right)^{(n)}\right\}_{n=1}^{N}$, with associated weights $\left\{w_{k}^{(n)}\right\}_{n=1}^{N}$. Since new particles are generated for every received symbol depending on the weight distribution of previous particles, we have introduced subscripts " $0: k$ " with $f$ variable to distinguish among the particles at each time step. We use SIS to build a recursive empirical approximation of a desired PDF, $p\left(s_{0: k}, f \mid y_{0: k}\right)$, by drawing samples from a different distribution (called the importance function) and assign appropriate normalized importance weights to these samples. According to SIS, the weights can be computed as

$$
w_{k}^{(n)} \propto \frac{p\left(s_{0: k}^{(n)}, f_{0: k}^{(n)} \mid y_{0: k}\right)}{\pi\left(s_{0: k}^{(n)}, f_{0: k}^{(n)} \mid y_{0: k}\right)}
$$

where the importance function, $\pi(\cdot)$, is chosen to admit a factorization of the form

$$
\begin{aligned}
\pi\left(s_{0: k}^{(n)}, f_{0: k}^{(n)} \mid y_{0: k}\right)=p\left(f_{k} \mid f_{0: k-1}^{(n)}, s_{0: k-1}^{(n)}, y_{0: k-1}\right) & \\
& \times p\left(s_{k} \mid s_{0: k-1}^{(n)}, f_{0: k}^{(n)}, y_{0: k}\right)
\end{aligned}
$$

By resorting to a recursive decomposition of the posterior distribution, the numerator in (5) can be expressed as

$$
\begin{aligned}
& p\left(s_{0: k}^{(n)}, f_{0: k}^{(n)} \mid y_{0: k}\right) \propto p\left(s_{k} \mid s_{0: k-1}^{(n)}, f_{0: k}^{(n)}, y_{0: k}\right) \times p\left(y_{k} \mid s_{k-1}^{(n)}, f_{k}^{(n)}\right) \\
\times & p\left(f_{k} \mid f_{0: k-1}^{(n)}, s_{0: k-1}^{(n)}, y_{0: k-1}\right) \times p\left(s_{0: k-1}^{(n)}, f_{0: k-1}^{(n)} \mid y_{0: k-1}\right)
\end{aligned}
$$

We can identify that two PDFs in (7) are the same as in (6). Substituting (7) and (6) in (5) and after some manipulation, we obtain

$$
w_{k}^{(n)}=w_{k-1}^{(n)} p\left(y_{k} \mid s_{k-1}^{(n)}, f_{k}^{(n)}\right)
$$

The algorithm can be described using following steps.

\subsection{Initialization}

We assume that the prior distribution of the transmitted symbol and frequency offset, $p\left(s_{-1}, f_{-1}\right)$, is known. The density of the frequency offset, $f_{-1}$, is considered to be uniform in the range $\left(-\frac{1}{2}, \frac{1}{2}\right)$. We assume that the first two symbols in the transmitted frame are known. The knowledge of the first symbol helps to avoid the use of differential modulation and provides the appropriate initial channel estimate, which is further tracked by kalman filtering. The assumption of the second symbol knowledge helps to avoid the $M$-fold ambiguity of the constellation and to acquire the full-range acquisition of the frequency offset. Note that the use of an initial pilot symbol or pilot space-time block transmission is common to many blind schemes to resolve such ambiguities $[9,10]$. Thus, we initialize the algorithm at time $k=-1$ as $s_{-1}^{(n)}=s_{-1}$ and $f_{-1}^{(n)} \sim \mathcal{U}\left(-\frac{1}{2}, \frac{1}{2}\right)$, $n=1,2, \ldots, N$. We initialize the weights of all the particles to be equal, i.e., $w_{-1}^{(n)}=1 / N$.

\subsection{Importance Sampling and Kalman Filtering}

The sampling from the importance function in (6) is accomplished in two steps. First, we obtain the frequency offset sample from $p\left(f_{k} \mid f_{0: k-1}^{(n)}, s_{0: k-1}^{(n)}, y_{0: k-1}\right)$. Following the approach in [11], we approximate this distribution by a beta distribution. Since beta distribution has the range $[0,1]$, we introduced a new frequency variable $\mathfrak{f}=f+0.5$, such that $\mathfrak{f}$ has the same range $[0,1]$. Thus, we draw the frequency offset sample from

$$
\mathfrak{f}_{k} \sim \beta\left(\mathfrak{f}_{k} ; U_{k}, V_{k}\right)
$$

where beta distribution parameters are $U_{k}=\overline{\mathfrak{f}}_{k}\left(\left(\overline{\mathfrak{f}}_{k}\left(1-\overline{\mathfrak{f}}_{k}\right) / \sigma_{\mathfrak{f}_{k}}^{2}\right)-\right.$ $1)$ and $V_{k}=\left(1-\overline{\mathfrak{f}}_{k}\right)\left(\left(\overline{\mathfrak{f}}_{k}\left(1-\overline{\mathfrak{f}}_{k}\right) / \sigma_{\mathfrak{f}_{k}}^{2}\right)-1\right)$, with $\overline{\mathfrak{f}}_{k}=$ $\sum_{n=1}^{N} w_{k-1}^{(n)} \mathfrak{f}_{k-1}^{(n)}$ and $\sigma_{\mathfrak{f}_{k}}^{2}=\sum_{n=1}^{N} w_{k-1}^{(n)}\left(\mathfrak{f}_{k-1}^{(n)}-\overline{\mathfrak{f}}_{k}\right)^{2}[11]$.

After drawing a carrier frequency offset sample, $f=\mathfrak{f}_{k}-0.5$, we draw a sample of the transmitted symbol from the second term of the proposal distribution in (6) i.e., $p\left(s_{k} \mid s_{0: k-1}^{(n)}, f_{0: k}^{(n)}, y_{0: k}\right)$. This sampling density can be rewritten as

$$
\begin{aligned}
p\left(s_{k}=S_{m} \mid s_{0: k-1}^{(n)}, f_{0: k}^{(n)}, y_{0: k}\right) & \\
& \propto p\left(y_{k} \mid s_{k}=S_{m}, s_{0: k-1}^{(n)}, f_{0: k}^{(n)}, y_{0: k-1}\right) \\
=\int_{\mathbf{h}_{k}} p\left(y_{k} \mid s_{k}=\right. & \left.S_{m}, f_{k}^{n}, \mathbf{h}_{k}\right) p\left(\mathbf{h}_{k} \mid s_{0: k-1}^{(n)}, f_{0: k}^{(n)}, y_{0: k-1}\right) d \mathbf{h}_{k}
\end{aligned}
$$

where $S_{m}$ is one of the possible transmitted symbol, $p\left(y_{k} \mid s_{k}=\right.$ $\left.S_{m}, f_{k}^{n}, \mathbf{h}_{k}\right)=\mathcal{N}\left(y_{k} ; h_{k} S_{m} e^{j 2 \pi f_{k}^{(n)} k}, \sigma_{w}^{2}\right), p\left(\mathbf{h}_{k} \mid s_{0: k-1}^{(n)}, f_{0: k}^{(n)}, y_{0: k-1}\right)=$ $\mathcal{N}\left(\mathbf{h}_{k} ; \boldsymbol{\mu}_{k \mid k-1}^{(n)}, \boldsymbol{\Sigma}_{k \mid k-1}^{(n)}\right)$ and $\mathcal{N}(\mathbf{x} ; \boldsymbol{\mu}, \boldsymbol{\Sigma})$ denotes the multivariate Gaussian pdf with mean $\boldsymbol{\mu}$ and covariance $\boldsymbol{\Sigma}$. We assume a Gaussian prior for the fading process, i.e., $\mathbf{h}_{-1} \sim \mathcal{N}\left(\mathbf{x} ; \boldsymbol{\mu}_{-1}, \boldsymbol{\Sigma}_{-1}\right)$. Note that the predictive channel mean

$$
\boldsymbol{\mu}_{k \mid k-1}^{(n)}=\mathbb{E}_{p\left(\mathbf{h}_{k} \mid s_{0: k-1}^{(n)}, f_{0: k}^{(n)}, y_{0: k-1}\right)}\left[\mathbf{h}_{k}\right]
$$

and the predictive channel covariance matrix

$$
\begin{aligned}
\boldsymbol{\Sigma}_{k \mid k-1}^{(n)}=\mathbb{E}_{p\left(\mathbf{h}_{k} \mid s_{0: k-1}^{(n)}, f_{0: k}^{(n)}, y_{0: k-1}\right)} & \\
& \times\left[\left(\mathbf{h}_{k}-\boldsymbol{\mu}_{k \mid k-1}^{(n)}\right)\left(\mathbf{h}_{k}-\boldsymbol{\mu}_{k \mid k-1}^{(n)}\right)^{H}\right]
\end{aligned}
$$

can be obtained in the closed form using a Kalman filter for each $n^{\text {th }}$ particle [12], current observation and the $n^{\text {th }}$ state trajectory $\left(s_{k}, f_{k}\right)^{(n)}$. Hence, integral in (10) can be solved to yield

$$
p\left(y_{k} \mid s_{k}=S_{m}, s_{0: k-1}^{(n)}, f_{0: k}^{(n)}, y_{0: k-1}\right)=\mathcal{N}\left(y_{k}, \bar{y}_{k}^{(n)}\left(S_{m}\right), \sigma_{y_{k}}^{2}\right)
$$

where $\bar{y}_{k}^{(n)}\left(S_{m}\right)=\mathbf{c}^{T} \boldsymbol{\mu}_{k \mid k-1}^{(n)} S_{m} e^{j 2 \pi f_{k}^{(n)} k}$ and $\sigma_{y_{k}}^{2}=\mathbf{c}^{T} \boldsymbol{\Sigma}_{k \mid k-1}^{(n)} \mathbf{c}+$ $\sigma_{w}^{2}$. Using (10) and (13), we can derive the probability mass function 
Table 1. Proposed Algorithm

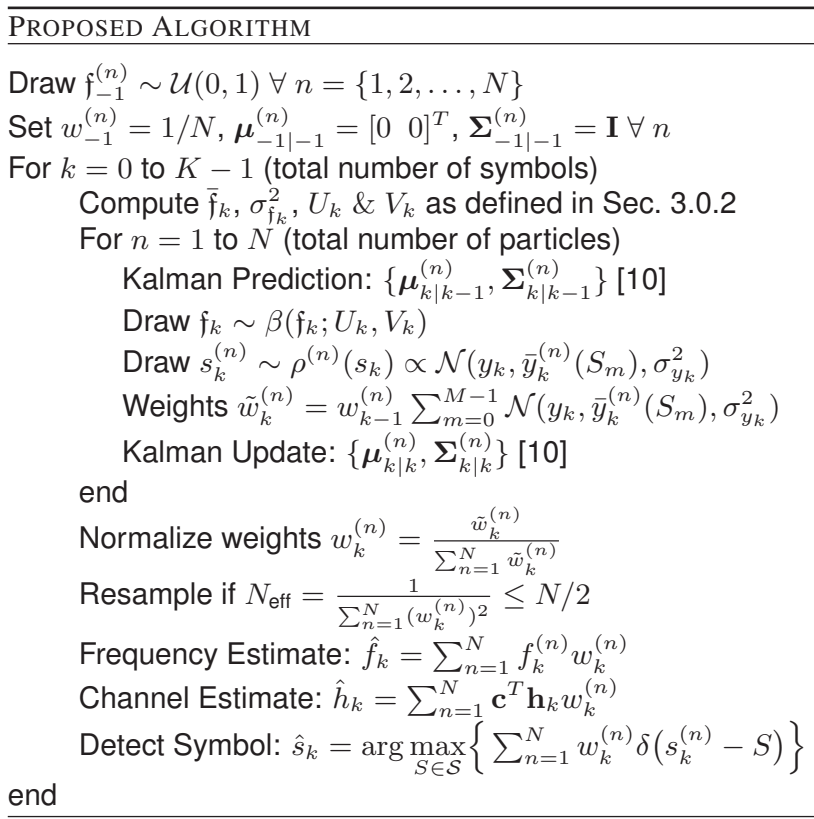

for each symbol of $\mathcal{S}$ as

$$
\begin{aligned}
\rho^{(n)}\left(s_{k}\right) & =p\left(s_{k}=S_{m} \mid s_{0: k-1}^{(n)}, f_{0: k}^{(n)}, y_{0: k}\right) \\
& =\frac{\mathcal{N}\left(y_{k}, \bar{y}_{k}^{(n)}\left(S_{m}\right), \sigma_{y_{k}}^{2}\right)}{\sum_{m=0}^{M-1} \mathcal{N}\left(y_{k}, \bar{y}_{k}^{(n)}\left(S_{m}\right), \sigma_{y_{k}}^{2}\right)}
\end{aligned}
$$

Hence, we draw the transmitted symbol according to

$$
s_{k}^{(n)} \sim \rho^{(n)}\left(s_{k}\right)
$$

\subsection{Weight update and Resampling}

After obtaining the new particles, we update their corresponding importance weights. Since all the possible transmitted symbols are equiprobable and current observation does not depend on the previous time states, given the current time state, we can rewrite the weight update expression (8) as

$$
\begin{aligned}
\tilde{w}_{k}^{(n)} & \propto w_{k-1}^{(n)} \sum_{m=0}^{M-1} p\left(y_{k} \mid S_{m}, s_{k-1}^{(n)}, f_{k}^{(n)}\right) \\
& =w_{k-1}^{(n)} \sum_{m=0}^{M-1} \mathcal{N}\left(y_{k}, \bar{y}_{k}^{(n)}\left(S_{m}\right), \sigma_{y_{k}}^{2}\right)
\end{aligned}
$$

where $\tilde{w}_{k}^{(n)}$ is the non-normalized importance weight for the $n$th particle. Finally, we normalize the weights as

$$
w_{k}^{(n)}=\frac{\tilde{w}_{k}^{(n)}}{\sum_{n=1}^{N} \tilde{w}_{k}^{(n)}}
$$

A well known problem in the practical implementation of the SIS algorithm is the degeneracy of the algorithm [13]. The common solution to this problem is to resample the particles. We consider resampling in our algorithm as described in [7].

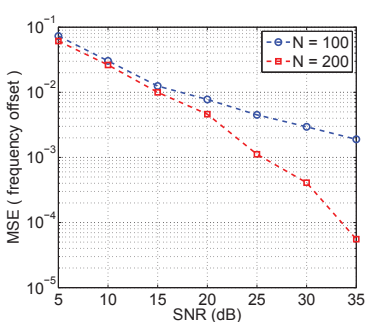

(a)

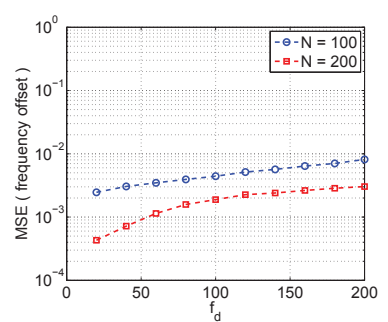

(b)
Fig. 1. Mean Square Error of frequency offset estimation (a) as a function of SNR (dB) with $f_{d}=22 \mathrm{~Hz}$. (b) as a function of doppler with $\mathrm{SNR}=30 \mathrm{~dB}$.

\subsection{Frequency Offset, Data and Channel Estimation}

The importance weights and the drawn samples for the carrier frequency offset are used to compute the Minimum Mean Square Error (MMSE) estimate, $\hat{f}_{k}$, of the true frequency offset as

$$
\hat{f}_{k}=\sum_{n=1}^{N} f_{k}^{(n)} w_{k}^{(n)}
$$

In addition, we compute the maximum a posteriori (MAP) estimates of the $k^{\text {th }}$ transmitted symbol as

$$
\hat{s}_{k}=\arg \max _{S \in \mathcal{S}}\left\{\sum_{n=1}^{N} w_{k}^{(n)} \delta\left(s_{k}^{(n)}-S\right)\right\}
$$

where $\delta(\cdot)$ denotes Dirac's delta function. Finally, the channel estimate at the $k^{\text {th }}$ instant is given by

$$
\hat{h}_{k}=\sum_{n=1}^{N} \mathbf{c}^{T} \boldsymbol{\mu}_{k \mid k}^{(n)} w_{k}^{(n)}
$$

The proposed algorithm is summarized in Table 1.

\section{SIMULATION RESULTS}

In this section, we present simulation results to verify the performance of our proposed algorithm. We consider a communication system with Binary Phase Shift Keying (BPSK) modulation and time-selective Rayleigh fading channel. The symbol time $T$ is assumed to be $10^{-4}$ seconds. The random frequency offset is assumed to be uniformly distributed in the range $(-0.48,0.48)$, i.e., approximately full acquisition range acquisition. The simulation results are averaged over $R=600$ Monte Carlo simulations, with each run consisting of a block of $K=100$ transmitted bits and $N=100$ and 200 particles respectively.

\subsection{Mean Square Error (MSE) of frequency offset estimation}

Fig. 1(a) shows the Mean Square Error (MSE) of frequency offset estimation for different values of SNR, averaged over $R$ simulation runs. We can see in Fig. 1(a) that the MSE decreases by increasing SNR and increasing the number of particles results in lower MSE value. The MSE is below $10^{-4}$ at $\mathrm{SNR}=35 \mathrm{~dB}$, which is acceptable result in the worst case scenario with frequency offset randomly distributed in the full acquisition range. Fig. 1(b) shows the Mean Square Error (MSE) of frequency offset estimation for different values of doppler at SNR $=30 \mathrm{~dB}$. The MSE increases by increasing doppler and increasing the number of particles results in lower MSE value as expected. 


\subsection{Bit Error Rate (BER) performance}

Fig. 2(a) shows the BER of the proposed receiver as a function of SNR. The reference curve in this figure, for the purpose of illustrating the slope of the BER curve, is the theoretical BER curve for BPSK in Rayleigh fading channel with no frequency offset error. We can see that there is a performance loss compared to the theoretical curve, e.g., an SNR of $35 \mathrm{~dB}$ is required to achieve a BER of $10^{-3}$ for $N=200$ particles which is approximately $11 \mathrm{~dB}$ from the theoretical curve. However we can see that slope of the simulated BER curve for $N=200$ particles is same as that of the reference curve which confirms the correct working of the proposed algorithm. The advantages of the proposed MKF receiver over existing non-MKF methods is discussed in Sec. 4.3.

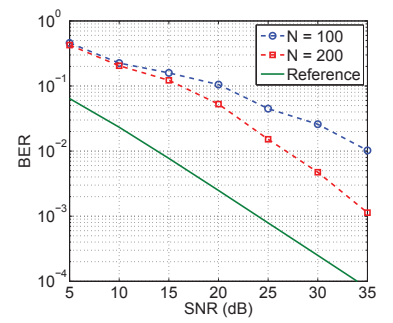

(a)

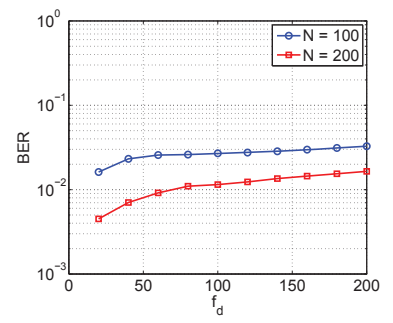

(b)
Fig. 2. Bit Error Rate of the proposed Receiver (a) as a function of SNR (dB) with $f_{d}=22 \mathrm{~Hz}$. (b) as a function of doppler with SNR $=$ $30 \mathrm{~dB}$.

The apparent loss in performance can be attributed to the fact that the carrier frequency offset is a very sensitive synchronization parameter. Even small residual errors in frequency offset estimation (of the order of 0.01) can lead to huge errors in data detection and bias the average BER results. The presented BER simulation results are unbiased results, i.e., we have not artificially discarded any initial bits to allow the algorithm to converge or $5 \%$ extreme results as suggested in [14]. Also note that the BER result in Fig. 2(a) corresponds to the worst case scenario with frequency offset randomly distributed in the full acquisition range.

Fig. 2(b) shows the BER of the proposed receiver as a function of doppler. The BER increases by increasing doppler and increasing the number of particles results in lower BER value as expected. Moreover, MSE in Fig. 1(b) and BER in Fig. 2(b) does not increase much by increasing the doppler, which shows the robustness of the algorithm also at higher doppler shifts.

\subsection{Comparison with Existing Frequency Offset Estimators}

The existing non-MKF based CFO estimators either assumes oversampling of the received signal by a factor of 8 , [4], or large number of symbols in the transmission frame, $(>500)$, $[4,5]$ or limited range of frequency offset estimation, $\left(\frac{1}{90}, \frac{1}{10}\right.$ or $\left.\frac{1}{60}\right)$ respectively $[1,4,5]$. We do not assume any oversampling and provide full range acquisition for frequency offset estimation. Moreover, our channel and frequency offset estimates converge quickly and does not require long transmission frames due to adaptive symbol by symbol processing. The above statements provide a brief comparison between the proposed scheme and existing non-MKF based CFO estimators.

\section{CONCLUSIONS}

In this paper, we have proposed a new blind algorithm for joint channel and frequency offset estimation, based on the combination of Kalman Filtering and Sequential Monte Carlo sampling. Our results have proved that MKF is a powerful tool for estimating unknown parameters in non-linear, non-Gaussian, real-time applications. We have studied the Mean Square Error (MSE) and Bit Error Rate (BER) performance of the proposed algorithm. The results show that BER of the proposed algorithm achieves the theoretical performance slope for the full acquisition range of carrier frequency offsets.

\section{REFERENCES}

[1] Y. Wang, K. Shi, and E. Serpedin, "Non data-aided feedforward carrier frequency offset estimators for QAM constellations: A nonlinear least-squares approach," EURASIP Journal on Applied Signal Processing, vol. 13, pp. 1993-2001, 2004.

[2] O. Besson and P. Stoica, "On frequency offset estimation for flat-fading channels," IEEE Commun. Lett., vol. 5, no. 10, pp. 402-404, Oct. 2001.

[3] M. Nissila and S. Pasupathy, "Joint estimation of carrier frequency offset and statistical parameters of the multipath fading channel," IEEE Trans. Commun., vol. 54, no. 6, pp. 10381048, Jun. 2006.

[4] F. Gini and G. B. Giannakis, "Frequency offset and symbol timing recovery in flat fading channel: a cyclostationary approach," IEEE Trans. Commun., vol. 46, pp. 400-411, 1998.

[5] E. Serpedin, A. Chevreuil, G. B. Giannakis, and P. Loubaton, "Blind channel and carrier frequency offset estimation using periodic modulation precoders," IEEE Trans. Signal Process., vol. 48, no. 8, pp. 2389-2405, Aug. 2000.

[6] R. Chen, X. Wang, and J. S. Liu, "Adaptive joint detection and decoding in flat fading channels via mixture Kalman filtering," IEEE Trans. Inf. Theory, vol. 46, pp. 2079-2094, Sep. 2000.

[7] T Ghirmai, N. F. Bugallo, J. Miguez, and P. M. Djuri, "A sequential monte carlo method for adaptive blind timing estimation and data detection," IEEE Transactions on Signal Processing, vol. 53, no. 8, pp. 2855-2865, Aug. 2005.

[8] M. J. Omidi, S. Pasupathy, and P. G. Gulak, "Joint data and Kalman estimation for Rayleigh fading channels," Wireless Presonal Commun., vol. 10, pp. 319-339, 1999.

[9] E. Beres and R. Adve, "Blind channel estimation for orthogonal STBC in MISO systems," IEEE Trans. Veh. Technol., vol. 56, no. 4, pp. 2042-2050, Jul. 2007.

[10] F. Z. Merli, X. Wang, and G. M. Vitetta, "A bayesian multiuser detection algorithm for MIMO-OFDM systems affected by multipath fading, carrier frequency offset, and phase noise," IEEE J. Sel. Areas Commun., vol. 26, pp. 506-516, Apr. 2008.

[11] T. Ghirmai, "Sequential Monte Carlo method for fixed symbol timing estimation and data detection," in Proc. IEEE Information Sciences and Systems, 2006.

[12] S. M. Kay, Fundamentals of Statistical Signal Processing: Estimation Theory, NJ: Prentice Hall, 1993.

[13] A. Doucet, S. Godsill, and C. Andrieu, "On sequential Monte Carlo sampling methods for bayesian filtering," Statistics and Computing, vol. 10, pp. 197-208, 2000.

[14] C. J. Bordin and M. G. S. Bruno, "Particle filters for joint blind equalization and decoding in frequency-selective channels," IEEE Transactions on Signal Processing, vol. 56, no. 6, pp. 2395-2405, June 2008. 